ISSN: 0210-7287

DOI: http://dx.doi.org/10.14201/1616201991534

\title{
LA LITERATURA MEDIEVAL DE LA PENÍNSULA IBÉRICA A LA LUZ DE UN COMPARATISMO FEMINISTA
}

\author{
Medieval Literature in the Iberian Peninsula \\ in the Light of a Feminist Comparatism
}

Laura PEREIRA DOMÍNGUEZ

Universidade de Santiago de Compostela

laura.pereira@usc.es

Recibido: junio de 2019; Aceptado: septiembre de 2019; Publicado: diciembre de 2019.

Ref. Bibl. LAURA PEREIRA DOMÍNGUEZ. LA LITERATURA MEDIEVAL

DE LA PENÍNSULA IBÉRICA A LA LUZ DE UN COMPARATISMO FEMINISTA.

1616: Anuario de Literatura Comparada, 9 (2019), 15-34

RESUMEN: En las últimas décadas, la literatura medieval ha llamado la atención de algunos comparatistas por el desafío que supone la realidad prenacional a una disciplina que concibe su labor en términos transnacionales. Se abre, así, la puerta el reconocimiento de otro tipo de relaciones entre literaturas agrupadas ya no solo en términos nacionales, sino en torno a otros ejes identitarios, como los que ha subrayado la crítica feminista en los últimos tiempos. Este trabajo se propone explorar las posibilidades de estudio de la literatura hecha por mujeres durante los siglos medievales en la península ibérica desde una perspectiva crítica feminista capaz de revisar las herramientas tradicionales de la literatura comparada.

Palabras clave: literatura comparada; autoría femenina; literatura medieval; literatura peninsular: historiografía feminista. 
ABSTRACT: In the last decades, medieval literature has attracted comparatists' attention due to the challenge that prenational reality poses to a discipline that conceives its work in transnational terms. In this way, it is possible to recognize different types of relations between literatures grouped not only in terms of national identity, but also derived from other identity axes, such as those that postcolonial and feminist criticism recently highlighted. In this paper, we propose the exploration of the possibilities of study of literature written by women during the medieval centuries in the Iberian Peninsula from a feminist critical perspective, with the tools of comparative literature.

Key words: Comparative Literature; Female Authorship; Medieval Literature; Peninsularism; Feminist Historiography.

\section{LA LITERATURA MEDIEVAL PENINSULAR Y LA LITERATURA COMPARADA}

La literatura medieval todavía no se ha podido encajar con éxito en el ámbito de la literatura comparada. Se han aducido diferentes razones que pueden explicar esta exclusión, constante desde los mismos inicios de la disciplina, y que apuntan hacia la concepción de los ejes que articulan los análisis comparatistas ${ }^{1}$. La literatura comparada aspira a trascender los límites entre literaturas, artes y otras disciplinas; y, en su origen, específicamente los límites nacionales impuestos para organizar las literaturas, como resultado del espíritu intelectual que rige el sistema académico desde el siglo XVIII. Estas fronteras corresponden a una idea de nación muy ligada a las lenguas y demarcan conjuntos de literaturas en base al poder identitario que se le atribuye a esta distinción ${ }^{2}$. En este contexto, la literatura medieval adquiere valor como lugar de origen de las literaturas nacionales y, por lo tanto, sus respectivas historiografías -el espacio del estudio de la literatura por excelencia todavía en la actualidad-incluyen en su repertorio los primeros textos creados en los estadios medievales de las lenguas después nacionales; ahora sí dentro del territorio delimitado por las fronteras de la nación moderna. Este salto anacrónico condiciona en este caso no solo la comprensión del objeto de estudio, sino también su creación.

Las literaturas medievales no tienen nación, si bien la crítica contemporánea accede a ellas desde una, necesariamente $-\mathrm{y}$, por supuesto, los

1. Sobre las primeras exclusiones de la literatura medieval en el marco de la literatura comparada, ver Domínguez (2008).

2. Ya Anderson (1993) señaló la importancia que, desde el siglo XVIII, las lenguas tuvieron en la configuración de la idea de nación. 
estudios medievales reconocen el carácter instrumental de dicha adscripción anacrónica-. Esta nación, sin embargo, si bien puede resultar útil (con todas las reservas explícitas) a la hora de emprender estudios diacrónicos, no ha gozado de la misma suerte en el campo de los estudios sincrónicos comparativos. Bien por la falta de una teorización estable y extendida de las fronteras culturales y políticas que rigen el mundo medieval ${ }^{3}$, bien por la poca importancia identitaria que a dicho criterio, aparentemente, concedieron las personas que habitaron la Edad Media, la literatura comparada no ha sido capaz de integrar la literatura medieval en sus modelos o, en todo caso, establecer un método para su estudio comparado.

Otro factor que se debe tener en cuenta al revisar esta omisión es el peso de la distribución institucional de los saberes, siempre señalado desde los debates alrededor de la literatura comparada como disciplina. En este caso ha contribuido a extender la creencia que liga la literatura comparada a la teoría literaria y que relega la labor de la historiografía literaria a los estudios de las literaturas nacionales y, con ellos, de las literaturas medievales. El resultado de este reparto paulatino es la exclusión prácticamente total de la literatura medieval de los últimos debates en el seno de la literatura comparada, que ponen en jaque, por un lado, la concepción de la historia literaria, pero también, por otro, los espacios de la literatura ${ }^{4}$.

Pero esta distinción tajante entre los intereses de la historiografía y de la literatura comparada no parece muy productiva en la práctica. Al contrario, las relaciones entre ambas disciplinas han sido muy fructíferas, al haber

3. Esta dificultad ha sido reconocida en el ámbito de lo global por redes de investigación como Defining The Global Middle Ages, entre cuyos objetivos están enmendar este vacío y ofrecer una definición de "lo global» válida para el mundo medieval. Sus propuestas en muchos casos se limitan, como ocurre con el campo de la literatura mundial, a una puesta en paralelo de diferentes objetos creados en diferentes lugares y momentos. Más interesantes son algunos intentos, dentro de esta línea, de esclarecimiento de relaciones efectivas entre culturas (intelectuales, obras, núcleos urbanos...) durante la Edad Media, para así aproximarse a la noción medieval de "global", cuya existencia se da por sentada. Sin embargo, el objeto observado y puesto en relación no abandona al fin y al cabo la esfera de lo tradicionalmente estudiado por diferentes disciplinas, a pesar de que gana novedad, muchas veces, gracias a la aplicación de metodologías habituales en la historiografía, como las redes sociales. Una dinámica similar es la de la literatura mundial, cuya apertura a un contexto mundo arrastró los objetos y las miradas sobre estos de las disciplinas que trataba de trascender.

4. Debido a su carácter instrumental, la recreación del mundo medieval relega a un segundo plano elementos como los espacios, los cuales no se pueden entender en términos nacionales y, por lo tanto, escapan a los métodos y fines de los estudios de área y, asimismo, de la literatura comparada. 
ayudado a revisar y modelar muchas de las categorías de análisis de la segunda. Recupero dos ejemplos que pueden ilustrar esta afirmación. En su artículo «Conjectures on World Literature» (2000), Franco Moretti recurría a los modelos de la historia económica para abordar las relaciones entre literaturas en un marco espacial mundo. En ese momento propone una nueva metodología, la distant reading, cuya mayor productividad se explica en un plano diacrónico. Un análisis distante debe ser capaz de revelar las disrupciones y continuidades en colecciones vastas de textos unidos por una genealogía idealmente amplia, aunque en la práctica se haya visto limitada por criterios lingüísticos en los que resuenan las concepciones nacionales tradicionales.

Algunas décadas antes, desde la ICLA (International Comparative Literature Association) se había promovido la redacción de historias comparadas de las literaturas, las cuales sirvieron de impulso renovador para la disciplina ${ }^{5}$. Las historias comparadas de la literatura proponen una organización de la realidad literaria similar a la que tradicionalmente se aplicó en el medievalismo. Frente al énfasis en la continuidad entre fenómenos literarios nacionales, se tiende a enfocar las literaturas que conviven en un espacio multilingüe, cuya máxima extensión es representada por el mundo. Este espacio se propone como un marco político, no necesariamente nacional, como la "Romania» para los siglos medievales, para cuya conformación el criterio espacial se cruza con uno lingüístico; o "Europa», cuya extensión la marcan relaciones políticas y no simplemente continentales ${ }^{6}$. No obstante, una diferencia esencial entre el medievalismo y la literatura comparada, aun cuando se refieran al mismo espacio, radica en el modo de concebir las relaciones entre diferentes conjuntos de literaturas. Especialmente durante los primeros años de las disciplinas, las relaciones en las que se fijó el medievalismo tendían a la filiación (basada en lenguas, esquemas compositivos u otros elementos del texto), mientras que la literatura comparada proponía modelos que permitiesen la comprensión de las diferentes redes que tejen estos conjuntos; un énfasis que de manera habitual fue productivo en el estudio de épocas modernas o contemporáneas.

El espacio que aquí nos compete, la península ibérica, ha sido estudiado por ambas disciplinas, pero de dos maneras muy distintas. Para los estudios medievales sirve como un espacio naturalizado, que funciona como

5. El resultado de este proyecto es la colección Comparative History of Literatures in European Languages (CHLEL), publicada por John Benjamins Publishing Company bajo la coordinación de Karen-Margrethe Lindskov Simonsen.

6. Un referente obligado en el análisis de las conexiones entre los espacios románico y europeo es el estudio de CurTius (1955). 
límite para las literaturas prenacionales que estudia, en cuya elección resuenan criterios nacionales o estatales. Por su parte, la literatura comparada ha empleado este espacio con el propósito contrario; esto es, para escapar de esta perspectiva nacional. Ambas disciplinas coinciden en señalar la importancia de la diversidad cultural en un espacio que, a fin de cuentas, es político y que no se corresponde con los límites nacionales en ningún caso.

La formulación del espacio ibérico como marco para el estudio de las literaturas medievales parte de una reconstrucción del pasado desde el presente, que termina por naturalizar un espacio determinado por el uso de las lenguas romances que hoy son oficiales en España (castellano, gallego, catalán) y desplaza a plano secundario otras lenguas de amplia utilización en los siglos medievales, como el hebreo o árabe. Esta separación se mantiene en los estudios medievales de la literatura, mientras que otras disciplinas que se encargan del análisis del mismo contexto (como la historia de la cultura, del arte o de la arqueología) no desatienden su integración. De este espacio surge la filiación académica que compondrá el Hispanismo y que se ha contestado desde la literatura comparada durante las últimas décadas?.

Desde una mirada comparatista, este espacio ibérico ha sido revisado como reacción a una concepción centralista de las literaturas portuguesa y española, tanto en su marco peninsular como en cuanto a sus relaciones con las culturas presentes en las antiguas colonias ${ }^{8}$. Gracias principalmente a los dos modelos hegemónicos en el estudio comparado de las literaturas peninsulares, es decir, aquellos que beben de la teoría de los polisistemas de Even-Zohar o de la teoría interliteraria de Durišin y que han sido enunciados desde la misma península ibérica (Pérez Isasi 2017, 351-355), se han podido superar los compartimentos estancos y monolingües que contenían los estudios de la literaturas nacionales dentro del marco peninsular, cuyas definiciones están determinadas por la coincidencia entre la lengua de sus textos y la oficial en sus territorios. Sin embargo, solo trascendiendo los límites de las literaturas nacionales, pertenezcan a la cultura hegemónica o a otras minorizadas, se podrá despegar el debate de la idea de nación y abandonar así «the ultimate Spanish nationalist game of hegemony: that of

7. El cuestionamiento del hispanismo como perspectiva integradora tiene sentido tras las críticas de corte multicultural y poscolonial, que ponen de relieve diversas carencias de las propuestas hispanistas (MORAÑa 2005).

8. Para un repaso crítico de los principales debates que se pueden agrupar bajo la categoría de "estudios ibéricos», tanto en la academia estadounidense como en la peninsular, ver PÉREZ IsASI (2017). 
problematizing the nation as the ultimate way to recenter all discussions under the master signifier of Spain» (Gabilondo 2014, 25).

Con todo, los resultados de estas propuestas siguen estando estructurados, a grandes rasgos, por la atención a ejes identitarios formulados en términos nacionales. Incluso la teoría interliteraria, según Duurišin (1984), se enuncia en este sentido y, es más, no solo no abandona el interés por los procesos en el nivel nacional, sino que los emplea como elemento de comparación en el proceso interliterario. No obstante, en esta misma teoría se apuntan nuevas secciones en espacios y comunidades que pueden servir, al menos idealmente, para organizar culturas en base a otros rasgos identitarios. Como vía de escape a este bucle ya casi definitorio de la literatura comparada, se plantea en este artículo una propuesta de estudio de un archivo de literatura hecha por mujeres ${ }^{9}$ durante la Edad Media, formulada desde una perspectiva crítica feminista que se sirva de categorías y metodologías comparatistas. La hipótesis de partida es que las comunidades interliterarias pueden pivotar sobre una identidad distinta a la nacional; en este caso, sexual o de género ${ }^{10}$.

Las comunidades interliterarias para Durišin son conjuntos supranacionales formados por literaturas nacionales interconectadas tras procesos históricos o literarios compartidos. Las comunidades suponen, entonces, un espacio intermedio entre la literatura individual o nacional y la general o mundial (Ďurišin 1984, 211) ${ }^{11}$. Es sugerente el planteamiento de esta noción, que permite afiliar (que no filiar) diferentes obras a un grupo

9. Entenderé por «literatura hecha por mujeres» todas aquellas obras en cuya creación las mujeres ocupan diferentes roles, desde poetas a mecenas, tal y como para la esfera del arte empleó la categoría Therese MARTIN (2015), al recuperar el sintagma medieval «mulier me fecit»: "One point of departure taken in this volumen is to refocus on the terminology used in the Middle Ages, particularly the verb "to make". For artist/patron is a false dichotomy, or, at least, a modern one [...]. This word [fecit] denotes at times the individual whose donation made the undertaking possible» $(2015,2)$. Martin mantiene la palabra inglesa maker en su discurso en español por la oportunidad que brinda de no determinar el sexo de "la persona que hace", decisión que, al ser ella la enunciadora, pone de relieve los prejuicios de los que ha partido la historia del arte al haber relacionado de manera preponderante a the makers con varones.

10. Parto conscientemente de una identidad sexual, como eje amplio, sin que ello vaya en detrimento de la interseccionalidad, ineludible en cualquier estudio que trate de mejorar los marcos existentes.

11. Esta noción la aplicó el mismo Ďurišin a la península ibérica, como espacio específico de relaciones entre las diferentes literaturas nacionales (portuguesa, gallega, española, catalana, vasca); propuesta en la que han profundizado CABo Aseguinolaza (2003), DomínGUEZ (2005) o PÉREZ ISASI (2017). 
después de que estas hayan participado de experiencias comunes. Igual que Ďurišin plantea esta hipótesis para las comunidades transnacionales, podemos imaginar conjuntos intermedios entre centros individuales y un nivel total superior que, en nuestra propuesta, abarcará el conjunto de literaturas de mujeres en la Edad Media. En su caso, los centros individuales no podrán ser ya nacionales, debido a que esta es una noción que no opera en las épocas premodernas y, por lo tanto, se deberán escoger diferentes unidades de análisis. En el otro extremo, el espacio superior observable tampoco se equiparará al mundo en los términos actuales, sino que deberá tomar su forma a partir de las relaciones que las sociedades mantenían entre sí.

\section{EL ESTUDIO DE LA LITERATURA HECHA POR MUJERES}

La propuesta de análisis de un conjunto de literatura hecha por mujeres como comunidad interliteraria responde a una observación del estado de los estudios medievales actuales. En este campo proliferan proyectos dedicados a mujeres generadoras de letras cuyos criterios a la hora de seleccionar y organizar el corpus son reveladores de un nuevo interés literario. Por un lado, este interés responde a una gran laguna que se percibía en el archivo académico de la literatura medieval, que se ha construido con una voluntad historicista, más interesada en explicar una identidad mediante el trazado de una genealogía cultural marcada por una sucesión teleológica de los hechos literarios hasta el presente desde el que se enuncia. En esta construcción de la literatura medieval se habían excluido diferentes textos en cuya génesis las mujeres jugaron un papel central, de manera similar a lo sucedido en los campos de la historia medieval y de la historia del arte. Por otro lado, en el plano teórico y epistémico, estos nuevos proyectos se pueden conectar con una alerta que se había detectado desde la crítica feminista de la segunda mitad del siglo Xx, la cual reconocía que las categorías hasta el momento manejadas no eran suficientes para entender la literatura hecha por mujeres. De entre todas las disciplinas que componen los estudios literarios, los resultados de esta preocupación teórica son especialmente atractivos para una corriente que basa su razón de ser en el rebase de fronteras, como es la literatura comparada: se resitúan, así, los límites de los cajones que contienen conjuntos literarios, en los cuales ya no tiene por qué primar un criterio nacional o, incluso, espacial.

La recolección y categorización de documentos medievales por parte de la historia de la literatura es en sí misma problemática. El diálogo entre 
el pasado y el presente que se establece en todo estudio histórico afecta siempre a las propias categorías; esto es, se aplican nociones operativas en los paradigmas actuales a una realidad pasada, en un salto que resulta habitualmente anacrónico a pesar de tratar de generar nociones más abarcadoras. Una de estas nociones es la de literatura. Bajo la etiqueta de "literatura medieval», se agrupan textos de diferente naturaleza: junto a la tradicional tríada archigenérica, se incluyen géneros como las crónicas o los tratados morales. Del mismo modo, otras categorías centrales para la crítica literaria cambian su definición para tratar de abarcar la concepción medieval correspondiente, como ocurre en el caso de la idea de "autoría" actual con respecto a las nociones de "auctor" $\mathrm{O}$ "auctoritas" ${ }^{12}$. La arbitrariedad implicada se hace explícita en estas clasificaciones gracias, en buena medida, a la alteridad que atraviesa no solo la experiencia lectora de los textos medievales, sino también su estudio. Este hecho facilita la identificación y revisión de sus categorías, primera acción que ha emprendido la crítica feminista.

Desde que el feminismo hizo notar que la experiencia histórica de las mujeres es distinta a la de los hombres, se ha pasado a aceptar el sexo/género como una categoría de análisis, es decir, se han empezado a establecer los conceptos y marcas que deben articular un estudio completo desde esta perspectiva. Se ha puesto de manifiesto, entonces, que los estudios de la cultura se han mantenido "dentro de las estructuras científico-sociales tradicionales, y han empleado antiguas formulaciones que sólo proporcionan explicaciones causales de carácter universal» (Scott 2008, 52); en las cuales, al contrario, se había dejado fuera a muchas otras existencias, en este caso, las de las mujeres. Tras negar dicha universalidad, se han propuesto nuevas perspectivas. La de Scott es una de las voces más influyentes dentro de este enfoque académico, de la que Judith Butler advirtió que "was not trying to make a claim for the simple inclusion of a consideration of women in the existing framework; rather she made the much stronger argument that the very framework depended upon that exclusion» (Butler 2011, 16). Si el marco que articula estos estudios está asentado en una perspectiva excluyente, se abren dos primeras opciones: cambiar el marco por otro incluyente o crear otro

12. La definición de auctor en la Edad Media se refiere tanto a la persona que hace una obra como a la que la impulsa de diferentes maneras. En ese sentido, el auctor puede ser quien idea la obra, como Alfonso x en el contexto de sus scriptoria (PUNDAY 2003, 31). Se ha señalado la importancia de la consideración de la noción de auctoritas al emprender estudios de interés comparatista, como los de traducción, por su relación con otras categorías, como auctor o traductor (BORSARI 2010). 
marco basado en la exclusión, en este caso, de esa perspectiva universal o masculina. Es esta una advertencia de gran calado, que también preocupó a otras críticas a la hora de reflexionar sobre su labor.

Lola Luna, en una ponencia que pronunció en 1995, avisaba de las dificultades que traía consigo la integración de una perspectiva feminista en un sistema de estudio machista, en el que sí existe un espacio para el estudio de las mujeres, si bien se reproducen en él los modelos tradicionales:

No nos engañemos, de poco sirve a una lectora ideal feminista esta segregación de sujetos y signos mujer si se continúa la adhesión a los valores simbólicos de los mundos representados y a los sentidos sexistas fijados por la crítica. Atención pues al mero análisis descriptivo de la imagen de la mujer sin una perspectiva crítica feminista, porque corremos el riesgo de perpetuar al infinito unas relaciones entre géneros que han sustentado históricamente el sistema de subordinación de la mujer (Luna 1996, 19-20).

Lo que Luna reclamaba para la historia de la literatura coincide con lo que Scott había demandado en el campo de la historia general: un estudio feminista de la cultura implica un cambio de paradigma, una atención al objeto desde una nueva óptica. Las categorías que empleamos en el estudio de la literatura medieval están determinadas, por lo tanto, por la mirada disciplinaria del presente. No solo eso, sino que también el repertorio disponible condiciona profundamente el estudio de la literatura, y este está archivado desde una perspectiva tradicional excluyente. Una mirada rápida a los grandes repertorios de literatura medieval revelará que los documentos archivables que agrupa corresponden a una autoría mayoritariamente masculina y que los pocos hechos por mujeres se han ordenado por criterios subjetivos similares a los genéricos, explicables por su relación heterónoma con respecto a la corriente que se considera principal; esto es, la escrita por hombres. El ejemplo más destacado de esta operación es el conjunto de textos conocido como la "Querella de las mujeres".

Desde la literatura comparada, la "Querella" ha sido caracterizada por Frank (2001) como una configuración interliteraria, noción que establece "by examining acts of writing in view of how authors responded to extant writings" (2001, 2), y más en concreto como el tipo de configuración que abarca "literatures that are, in theory, equal and compete with each other" $(2001,6)$. No debemos pasar por alto la llamada de atención que desliza Frank en su definición, y es que, si bien estos grupos de literaturas son en teoría iguales, en la práctica existe una jerarquía claramente marcada. En el caso de la "Querella", destaca su poca presencia en los cauces académicos 
y educativos habituales, de ningún modo comparable con otros géneros o conjuntos de textos en el mismo contexto ${ }^{13}$.

La "Querella» es excepcional. No se han establecido otros grandes grupos para literaturas de mujeres anteriores al siglo XIX y, quizá por esta razón, su definición ha sido ejemplar para otros proyectos. Las coordenadas espaciotemporales de la "Querella" son amplias: bajo esta etiqueta, se agrupan textos que se escriben y circulan por diferentes territorios europeos desde finales del siglo XIV hasta el siglo XVIII. Además, estas coordenadas espacio-temporales no condiciona una organización interna; esto es, al contrario de lo que sucede en el estudio de otros conjuntos, como los genéricos, no prima un criterio teleológico o sucesivo en su disposición. La "Querella" se presenta como una constelación yuxtapuesta de testimonios escritos, fruto de una actitud compartida, específicamente representada por el deseo de equiparación del sexo femenino y masculino. Las categorías más básicas desde las que se establece el conjunto pueden guiarnos en el examen de los proyectos de investigación propuestos para estudiar literatura hecha por mujeres, ya que esta recolección y disposición de hechos literarios tiene consecuencias epistemológicas de gran calado, tanto desde una perspectiva feminista como comparatista.

En los últimos años se han emprendido varios proyectos de investigación que tienen como objetivo el estudio de la literatura escrita por mujeres; centrados, en gran medida, en llenar los vacíos que la crítica tradicional había dejado. Podemos tomar como ejemplo cuatro grandes proyectos de amplia difusión: Bieses (UNED, España), Women Writers (Heygens ING, Pa1́ses Bajos), Escritoras (Universidade de Lisboa, Portugal) e Italian Women Writers (University of Chicago, Estados Unidos) ${ }^{14}$. El resultado son bases de datos que tratan de registrar todo lo actualmente accesible, conformando un repertorio de literatura hecha por mujeres. Al observar los criterios de selección del corpus de estas bases de datos, es posible delimitar cómo se está creando un archivo, entendiendo esta noción como «fundamentally a matter of discrimination and of selection, which, in the end, results in the granting of a privileged status to certain written documents, and the refusal

13. En este sentido, es significativo el peso que la editorial de la Asociación Cultural Almudayna tiene en la divulgación de las investigaciones acerca de la Querella de las mujeres en España.

14. Las bases de datos resultantes de estos proyectos son de acceso libre y se pueden encontrar en los siguientes portales: Bieses está en http://www.bieses.net/ [24 abril 2018], WomenWriters, en http://neww.huygens.knaw.nl/ [24 abril 2018], Escritoras, en http:// www.escritoras-em-portugues.eu/ [24 abril 2018] e Italian Women Writers, en https://www. lib.uchicago.edu/efts/IWW/ [24 abril 2018]. 
of that same status to others, thereby judged "unarchivable". The archive is, therefore, not a piece of data, but a status" (Mbembe 2002, 20). Estas bases de datos crean, en última instancia, la idea de literatura hecha por mujeres.

Elimino de la perífrasis el adjetivo "medieval», ya que una de las características de estos proyectos, que coincide con el establecimiento de la "Querella», es la difuminación de la apreciación cronológica característica de las historias tradicionales. Bieses, base de datos que recoge referencias bibliográficas sobre escritoras, amplía su selección a las creadoras que desempeñaron su actividad literaria antes del siglo XIX. También WomenWriters y Escritoras establecen como criterio que las obras fuesen escritas antes del 1900, cronología ampliada hasta 1945 en el caso de Italian Women Writers. Parece que esta decisión tiene que ver con las lagunas dejadas por la historia menos atenta a la creación de mujeres, que dificultan la creación de un conjunto nutrido de esta literatura, necesario para emprender un estudio amplio. Las dificultades cuantitativas a la hora de establecer un corpus representativo pueden ser una otro razón motivo que explique la ampliación los marcos cronológicos a varios siglos, como ocurría con la "Querella». Esta amplitud, además, favorece una sensación de naturalidad, de lógica de bases de datos en la que se incluye todo lo accesible bajo la apariencia de una falta de orden y jerarquía.

Ya Claudio Guillén había apuntado que las unidades temporales extensas que permiten estructurar la historiografía literaria son flexibles y no se limitan a un ámbito nacional $(1985,362)$. Salvo algunas excepciones locales, estas unidades se presentan, en cierto modo, como universales al ser propuestas para ordenar los tiempos históricos en un nivel supranacional. Se pueden proponer diferentes críticas a esta propuesta, argumentando la existencia de temporalidades particulares para diferentes naciones u organizaciones del tiempo acordes a diferentes identidades no nacionales. Es este el caso de Knibiehler, quien señaló en su trabajo clásico Une bistoire des femmes est-il possible? (1984) la existencia de una cronología propia para las mujeres -con todo, relacionada con la masculina-:

Les séries chronologiques masculines ont leur cohérence, leur orientation, leur perspective. Les chronologiques féminines proposent une autre perspective, qui a pu d'abord paraitre déconcertante, ou dépourvue de sens, dans les hauts lieux où l'on écrit l'histoire. C'est sans doute la raison du ghetto initial: «l'institution", on l'a dit, a laissé l'histoire des femmes se développer à part, parallèlement, sans dialogue (Knibiehler 1984, 52-53).

Hablar de series cronológicas masculinas y femeninas implica reconocer lo arbitrario de la concepción temporal en el marco de la historia. Los cortes y la ordenación de las obras en una línea cronológica son algunas 
de las construcciones más reificadas de la historia de la literatura, cuya superación no parece sencilla. El archivo o, incluso, la historia de las mujeres debería regirse, entonces, por sus propios momentos, acordes al desarrollo de la literatura hecha por mujeres (si se mantiene un espíritu teleológico) o acordes a otro tipo de criterios que se desprendan de la interpretación de cada escenario concreto. Como la misma Knibiehler amplía, esta opción no parece, sin embargo, realista:

Mais il est bien évident que le ghetto est invivable: une chronologie purement "féminine" est impensable; elle ne devient intelligible que si l'on établit des tabels synchrones pour faire apparaitre des corrélations avec l'histoire, politique, économique, culturelle. Ces corrélations confirment la dépendance, le manque d'initiative des femmes. Mais elles appellent aussi une autre remarque: les séries féminines sont décalées. C'est peutêtre là un aspect de ce qu'Althusser appelait la "temporalité différentielle» (Knibiehler 1984, 53).

La dimensión institucional del estudio de la literatura hecha por mujeres la obliga a relacionarse con otras series cronológicas -también masculinas-, tanto pertenecientes al mismo ámbito de la literatura como a otros, tales como los señalados por Knibiehler de apariencia superior y articuladora -por ejemplo, la historia cultural o económica-. Esto explica que se mantengan en el horizonte algunas marcas temporales -y, podríamos añadir, también espaciales- que orientan el orden de una manera tradicional. La principal consecuencia es la conservación de estos aspectos como marcos asépticos, naturalizados, sobre los que descansan los tiempos, tanto en sentido cronológico como periodológico.

Los períodos literarios están al margen de estos nuevos proyectos, en tanto son considerados como "auténticos fósiles conceptuales» -tal y como los ha definido Domínguez $(2004,122)$ - debido a que "rara vez son reconocidos en términos de sus funciones heurísticas» (Domínguez 2004, 122). Se entienden como categorías cosificadas, inevitables y comprometidas con una ordenación sucesiva del devenir histórico. Las bases de datos que arriba mencionamos se desprenden de este corsé temporal, ya que no pretenden adscribir las nuevas obras a un período ya existente -que reconocemos como criterio subjetivo de clasificación masculina, que va más allá de lo temporal para cruzarse con elementos estilísticos o genológicos-.

Asimismo, estos modelos delimitan el espacio, otro de los puntos clave en la selección de las literaturas, según criterios distintos a los tradicionales. Bieses e Italian Women Writers siguen un modelo nacional, el cual, en el contexto de una base de datos, parece intentar renovar la pretensión de neutralidad de ese tipo de herramientas. Quizá tenga esto que ver con la segunda paradoja que señaló Anderson $(1993,22)$, que conduce a 
reconocer lo nacional como categoría sociocultural universal, casi natural hoy en día. Tanto Bieses como Italian Women Writers emplean los límites de los estados actuales como índice de localización de las escritoras: digamos que se señala su localización en un mapa físico al que se superpone el mapa político actual. De un modo similar, Women Writers amplía su alcance a Europa, marco que arrastra consigo las mismas reservas en cuanto a los límites políticos anacrónicos.

Si bien las categorías temporales que tradicionalmente ordenaban las relaciones literarias (junto a, por ejemplo, la periodología o la genología) pierden importancia crítica en las bases de datos, no se puede decir lo mismo con respecto al factor nacional. En este punto se pueden reconocer deudas contraídas con los estudios literarios clásicos. Esto es especialmente visible en Bieses, que reproduce en sus criterios de selección los que serían propios del hispanismo más sesgado; algo que concuerda con el punto de partida de la base de datos, un catálogo publicado entre 1903 y $1905^{15}$. En la base de datos, ampliada por el equipo de investigación, se reúnen referencias bibliográficas sobre mujeres que escribieron en lo que hoy es el Estado español y principalmente en castellano; aunque se incluyen autoras como Isabel de Villena, que lo hizo en catalán. De manera similar, Escritoras sigue un criterio lingüístico cruzado con el nacional que le permite seleccionar todas aquellas mujeres que escribieron en portugués en los territorios regidos por los gobiernos portugueses, tanto en lo que hoy es Portugal como en los espacios ocupados durante las colonizaciones. Por su parte, Italian Women Writers y Women Writers favorecen una comprensión del espacio en otros términos. La primera incluye escritoras en el territorio que hoy ocupa Italia, dividido a su vez en las regiones actuales. En la misma línea, WomenWriters emplea las fronteras estatales establecidas hoy en Europa para organizar su repertorio, del que después indica la lengua de escritura.

Propongo que estas fronteras estatales, al contrario de lo que ocurre en el caso de las organizaciones tradicionales de la literatura, no están articulando conjuntos operativos en el estudio de la literatura hecha por mujeres, sino que componen un espacio instrumental en estas fases iniciales del archivo. Lo que me lleva a realizar dicha afirmación es la orientación de las bases de datos como herramienta de búsqueda y no como relato en estos momentos. Los Estados parecen ocupar aquí una función instrumental, con

15. En la misma presentación del proyecto, se identifica como punto de partida el catálogo Apuntes para una biblioteca de escritoras españolas desde el año 1401 al 1833, de Manuel Serrano y Sanz. 
el peligro de asunción de neutralidad que esto implica. Puesto que este criterio no puede servir en este caso para adscribir obras y escritoras a una identidad nacional: y es que no solo no forman parte del canon, sino tampoco del archivo nacional.

\section{3. ¿UNA HISTORIA DE LITERATURA DE MUJERES?}

La finalidad de este tipo de estudios no tiene por qué estar ligada a una genealogía nacional, algo que demuestra la nula presencia de este repertorio en circuitos educativos reglados, así como en bibliotecas y archivos públicos ${ }^{16}$. Por el contrario, la marca de grupo es el sexo/género. A este respecto resulta interesante la declaración de intenciones del último estudio de Classen, Reading medieval European women writers:

[My intentions are] to assemble all the relevant information and to develop a better understanding of some of their most important narratives and thus to gain a solid cultural-historical comprehension of women's actual participation in the culture of their time across medieval Europe and how they managed to find a literary voice on their own (Classen 2016, 9).

El objetivo pasa por la refundación histórico-cultural del papel de las mujeres, a partir de una mirada transnacional que de hecho lleva a Classen a afirmar, creo que de una manera bastante arriesgada, que "we can be certain that our conclusions will allow numerous implications for female writers in the Arabic or Asian societies during that period as well» (Classen 2016, 14).

Si bien los criterios seleccionados en las bases de datos satisfacen sus objetivos en cuanto archivos, la integración del conocimiento de estas autoras y sus obras en el conjunto de la investigación académica obliga a desprenderse de la constelación paratáctica para trazar un relato en términos similares a los existentes en otros ámbitos de estudio. Es esta la interacción entre disciplinas a la que hacía referencia Knibiehler cuando abordó la cronología. También en este contexto Foucault (2002) señaló la relación entre el saber y el poder, que nos conduce a la asimilación de los códigos externos que ordenan nuestro relato. Antes de valorar la conveniencia del

16. A este respecto es llamativa la lucha de la Biblioteca de Mujeres en Madrid, similar a otros casos de colecciones de literatura de mujeres (CARDO 2018) o informes como el de LóPEZ-NAVAJAS (2014), que evalúa la presencia y el tratamiento de escritoras en manuales de educación secundaria. 
empleo de la historia y de sus categorías para los nuevos propósitos debe realizarse una revisión en estos términos de los discursos en juego que evite su naturalización.

El salto de la constelación paratáctica a la historia revelaría su voluntad de explicar una identidad, de proporcionarle una genealogía y una razón de ser, igual que la historia de la literatura tradicional escondía y reforzaba un Geist. Sin embargo, es posible trazar otro tipo de cortes temporales sin perseguir que guarden entre ellos una relación teleológica y alejarse así, en cierta medida, de las categorías tradicionales, al mismo tiempo que se permite la interacción con las explicaciones culturales hegemónicas. En este punto, la noción de comunidad interliteraria, cruzada con un criterio cronológico, resulta útil.

La comunidad interliteraria permite articular el conjunto de la literatura hecha por mujeres en diferentes niveles y de maneras distintas ${ }^{17}$. Dentro de ese nivel se reconocen conjuntos más pequeños capaces de establecer relaciones, tanto de una forma interna (entre ellos), como externa (con otros). Del mismo modo, es posible trazar conjuntos cada vez más grandes, marcados por las relaciones en la misma comunidad, hasta una extensión mundo. Asimismo, a la hora de disponer relaciones con otras categorías de los estudios literarios tradicionales organizados por unidades espaciales y temporales, como los períodos o las naciones que arriba mencionamos, los cortes propuestos por las bases de datos resultan operativos.

Propongámonos ahora relacionar la literatura medieval peninsular con la comunidad interliteraria de mujeres ${ }^{18}$. El período medieval se define desde aquel deseo universalista que Scott (2008) denunciaba, el cual tiene claras implicaciones hermenéuticas, como recordaba Domínguez (2004), ya que excluye la experiencia de las mujeres ${ }^{19}$. Por esto es preciso recurrir a otros cortes temporales instrumentales -también arbitrarios- como pueden ser los siglos ${ }^{20}$. El mismo corte en el eje espacial cumpliría la misma

17. Sobre estas relaciones interliterarias concebidas en términos naciones, véase D̃URIŠIN (1984 y 1989).

18. Nótese que no se parte de un grupo previamente relacionado, cuyos vínculos deben ser descubiertos, sino que se está presentando un gran conjunto solamente atravesado por una identidad sexual, cuyas conexiones son múltiples e, incluso, puede que no existan.

19. Esta crítica no solo es válida para la identidad femenina, como en su momento pusieron de relieve otras propuestas críticas, como la poscolonial.

20. Es reveladora, a este respecto, una de las decisiones habituales en la historiografía, que también escapa de la homogeneización que resulta de categorías periodológicas, como la división temporal por reinados o gobiernos de diferentes figuras. Desde una perspectiva interliteraria, por su parte, GUTIÉRREZ GARCíA (2004) examinó la dificultad de señalar fechas clave en una comunidad interliteraria. 
función. Acotemos, entonces, los límites a las literaturas en el siglo XV en la península ibérica.

Los espacios peninsulares en el siglo XV se conforman desde una perspectiva feminista de una manera distinta a la tradicional, ya que deben ser capaces de reflejar la movilidad propia de las mujeres, especialmente las pertenecientes a las clases dominantes, que son también las que mayoritariamente creaban y leían o escuchaban literatura. En este sentido, es preciso tener en cuenta que dentro de las funciones políticas de las mujeres los acuerdos matrimoniales gozan de una posición destacada. Esta movilidad tendrá implicaciones significativas en la conformación lingüística de la literatura que circulará con ellas, además de los lazos personales entre diferentes comunidades humanas, que pueden funcionar como núcleos de la comunidad interliteraria.

Una vez más, la literatura comparada puede recurrir al faro de la historia para reconocer sus unidades relacionales. A pequeña escala, propongo dos tipos de unidades útiles para el estudio de la literatura hecha por mujeres en el siglo XV: los círculos de mecenazgo y las comunidades de lectoras. Los círculos de mecenazgo se organizan alrededor de una figura femenina que comisiona obras literarias, ya sea de hombres, ya de mujeres. Estos círculos cuentan con una nómina de obras, habitualmente en diferentes idiomas, que pueden estar relacionadas con otros círculos, bien por la dependencia múltiple de los escritores o escritoras, que pueden participar en diferentes círculos al mismo tiempo, bien por las relaciones entre mecenas. En el siglo xv es posible recuperar el ejemplo de Isabel de Portugal (14321455), que introduce la obra de Christine de Pizan en la península gracias al envío de un libro por parte de su tía Isabel (1397-1471), también gran promotora de la cultura en el ducado de Borgoña, territorio que gobernaba junto a su marido. Relaciones similares se pueden establecer para el caso de los trasvases entre el círculo cultural de Isabel I de Castilla (1451-1504) y sus hijas, quienes dejarán su lugar de origen por razones de política matrimonial y para las que, por lo tanto, un marco de estudio peninsular no sería suficiente ${ }^{21}$. Las comunidades de lectoras, por su parte, tienen especial relación con las comunidades religiosas. A pesar de que todavía hoy los datos son difícilmente accesibles, debido al carácter íntimo de los espacios en que tuvieron lugar, los conventos como espacios de creación artística e

21. La biblioteca de la reina Isabel I de Castilla ha sido una de las que más interés ha despertado en el contexto medieval. Se puede consultar el volumen de RUIz GARCía (2004), en el que no solo se detallan los volúmenes que aquella biblioteca seguramente contenía, sino también algunos de los libros que la reina hizo llegar a sus hijas como presentes nupciales (ver Ruiz GARCía 2004, 56; MARTínez AlCORLo 2016, 170). 
intelectual conforman el núcleo de varias investigaciones ${ }^{22}$. Estas unidades, en la medida en que se sitúan en un espacio concreto, pueden relacionarse con otras que también lo ocupen -como lo hacen los reinos, las naciones, los estados- y, al mismo tiempo, no perder su especificidad.

La teoría interliteraria ofrece nuevas herramientas para la comparación de diferentes literaturas, ya que las hasta ahora existentes se habían revelado demasiado homogeneizadoras; si bien es cierto que toda abstracción implica un cierto grado de homogeneización. Frente a nociones que ya cargan una mochila ideológica importante, como la de nación, Ďurišin propone la creación de diferentes cortes espaciales que permitan enfocar diferentes conexiones literarias. Del mismo modo, una mayor flexibilidad en la delimitación de unidades espaciales de distinta magnitud facilita una comprensión de la realidad literaria de mujeres en los términos expuestos y propicia el rastreo de los flujos que conectan diferentes conjuntos literarios, de un modo más próximo al contexto de creación y recepción de las obras.

Junto al cambio de unidades temporales y espaciales, en la comunidad interliteraria de mujeres caben otros tipos de categorías organizativas subjetivas capaces de satisfacer las funciones que en la crítica tradicional cumplen, por ejemplo, los géneros y movimientos. De esta manera podría entenderse la "Querella» tal y como está formulada. El cambio de perspectiva en la categoría reside en su definición, no por rasgos intrínsecos habituales, como estilísticos o generacionales, sino por una actitud hacia otros textos, unificada por la crítica. Asimismo, la división genérica y las relaciones entre los géneros literarios merecen una revisión en este contexto. Así, formas como las epístolas, que conforman un modo habitual de expresión femenina, deberán resituarse en el sistema genérico de literatura hecha por mujeres. El tipo de elementos, su jerarquía (en el caso de haberla) y sus modos de relación deberán establecerse como resultado de un estudio del conjunto literario que se quiere analizar. Es fácil advertir que las unidades de análisis propuestas aquí, igual que otras que se podrán formular para otros casos, no son estables a lo largo del tiempo. Resulta difícil todavía en este momento perseguir la estabilidad en un conjunto excluido de las instituciones que sí han ordenado otros.

22. En las últimas décadas, de la mano de la historia de la lectura de las mujeres, se han bosquejado comunidades de lectoras y creadoras, tanto religiosas como laicas, que también se pueden realizar espacios físicos. En el ámbito específicamente literario, se pueden mencionar los proyectos «Medieval Convent Drama», de la Universidad de Fribourg (ROBINSON en prensa), O «Teatro y academias", de la Universidade de Santiago de Compostela, desarrollado por Paula Casariego CastiÑeiras (Contrato Posdoutoral Xunta de Galicia 2017). 
En definitiva, la creación de una historia de las literaturas hechas por mujeres corre el riesgo de responder a una asimilación de las categorías masculinas o tradicionales de creación y organización del conocimiento literario (tales como "nación" o "lengua»). A la vista de las críticas desde la historiografía feminista y de los marcos empleados en nuevos procesos de archivo de literatura hecha por mujeres, podemos preguntarnos si realmente es productivo prescindir de modelos de estudio más maleables que sean capaces de abarcar las particularidades de estos conjuntos literarios. Al mismo tiempo, no se puede olvidar que la historia es uno de los modos de construcción del saber más autorizados en la actualidad, por lo que cualquier esquema de estudio de literatura hecha por mujeres debe ser capaz de dialogar con sus categorías sin, al contrario, fundirse en ellas. Esta tensión está lejos de ser resuelta, pero no por ello debe dejar de nombrarse.

\section{Conclusiones}

La literatura comparada, una disciplina que se ha caracterizado por un autocuestionamiento continuo, proporciona fórmulas que, como mínimo, motivan o alumbran aspectos candentes capaces de abrir la puerta a nuevos encajes de realidades literarias en la crítica académica. A lo largo de este breve ensayo, he querido hacer alusión a estos puntos de análisis desde una idea de literatura separada de la tradicional, con unas redes de influencia particulares, una noción de autoría diferente e, incluso, un inventario propio de géneros.

En este repaso se han alterado muchas de las características propias de la crítica tradicional, excepto una: su calidad de excluyente. A fin de cuentas, se han propuesto herramientas propicias para un tipo de creación concreta, limitada. Si miramos este modelo con una voluntad incluyente, podemos preguntarnos qué puede aportar una comunidad interliteraria de mujeres a la literatura comparada. Y seguramente la respuesta sea flexibilidad, que puede, incluso, ser extensiva. La creación de comunidades basadas en otros rasgos identitarios, no nacionales, puede contribuir a un reajuste de las categorías y, por lo tanto, llevarnos a comprender desde prismas todavía inestables la interacción de agrupaciones literarias. 


\section{REFERENCIAS}

ANDERSON, Benedict. 1993. Comunidades imaginadas: reflexiones sobre el origen y la difusión del nacionalismo. Traducido por Eduardo L. Suárez. México: Fondo de Cultura Económica.

BORSARI, Elisa. 2010. "Auctor y auctoritas: apuntes sobre la traducción de los clásicos durante la Edad Media». En Actas del XIII Congreso Internacional de la Asociación Hispánica de Literatura Medieval (Valladolid, 15 a 19 de septiembre de 2009): in memoriam Alan Deyermond. Editado por José Manuel Fradejas Rueda et al., 455-467. Valladolid: Universidad de Valladolid.

BuTLER, Judith. 2011. «Speaking up, Talking Back». En The Question of Gender. Joan W. Scott's Critical Feminism, editado por Judith Butler y Elizabeth Weed, 1128. Bloomington: Indiana University Press.

Cabo Aseguinolaza, Fernando. 2003. "Geography and Literature: On a Comparative History of the Literatures in the Iberian Peninsula". Neobelicon 30 (1): 117-125.

CARDO, Yolanda. 2018. "Biblioteca de Mujeres de Madrid. La cruzada personal de Marisa Mediavilla». Crónica Global. El Español, 25 de febrero. Web.

Classen, Albrecht. 2016. Reading Medieval European Women Writers: Strong Literary Witnesses from the Past. Frankfurt/Nueva York: Peter Lang.

CuRTIUS, Ernst Robert. 1955. Literatura europea y Edad Media latina. Traducido por Margit Frenk y Antonio Alatorre. México: Fondo de Cultura Económica.

Domínguez, César. 2004. "Periodología, cambio literario e historia comparada: apuntes metodológicos». En Bases metodolóxicas para unha bistoria comparada das literaturas na Península Ibérica, editado por Anxo Abuín González y Anxo Tarrío Varela, 121-152. Santiago de Compostela: Universidade de Santiago de Compostela.

Domínguez, César. 2005. "Os horizontes da teoría interliteraria na Península Ibérica: recepción e campo de probas». Boletín galego de literatura 34: 37-65.

Domínguez, César. 2008. "Las literaturas medievales como provocación de la literatura comparada. Reflexiones sobre las formaciones culturales no-nacionales». Revista de Poética Medieval 20: 99-126.

ĎuRIŠIN, Dionýz. 1984. "Specific Interliterary Communities». Neohelicon 11: 211-241. ĎurišIn, Dionýz. 1989. Theory of Interliterary Process. Bratislava: Veda.

Foucault, Michel. 2002. Vigilar y castigar. Nacimiento de la prisión. Traducido por Aurelio Garzón del Camino. Buenos Aires: Siglo XXI.

Frank, Armin Paul. 2001. "On the Comparison of Interliterary Configurations". CLCWeb: Comparative Literature and Culture 3.2. Acceso el 20 de abril de 2018. https://doi.org/10.7771/1481-4374.1118.

GABILONDO, Joseba. 2013-2014. "Spanish Nationalist Excess: A Decolonial and Postnational Critique of Iberian Studies». Prosopopeya 8: 23-60.

Guillén, Claudio. 1985. Entre lo uno y lo diverso. Barcelona: Crítica.

GuTiÉRREZ GARCíA, Santiago. 2004. «Periodización y fechas clave. Una aproximación a la historiografía comparada de las literaturas ibéricas». Bases metodolóxicas para unha historia comparada das literaturas na Península Ibérica, editado 
por Anxo Abuín González y Anzo Tarrío Varela, 153-180. Santiago de Compostela: Universidade de Santiago de Compostela.

KNIBIEHLER, Yvonne. 1984. "Chronologie et histoire des femmes". En Une histoire des femmes est-elle possible?, editado por Michelle Perrot. París: Rivages.

LóPEZ-NAVAjAs, Ana. 2014. "Análisis de la ausencia de las mujeres en los manuales de la ESO: una genealogía de conocimiento ocultada». Revista de Educación 363: 282-308.

LunA, Lola. 1996. "Leyendo como una mujer la imagen de la mujer». En Leyendo como una mujer la imagen de la mujer. Barcelona: Anthropos.

MARTIN, Therese. 2012. Reassessing the Roles of Women as "Makers» of Medieval Art and Architecture. Leiden: Brill.

MARTíNeZ ALCORLO, Ruth. 2016. La literatura en torno a la primogénita de los Reyes Católicos: Isabel de Castilla y Aragón, princesa y reina de Portugal (14701498). Tesis doctoral. Universidad Complutense de Madrid.

MBEmBe, Achille. 2002. "The Power of the Archive and its Limits». En Refiguring the Archive, editado por Carolyn Hamilton, 19-26. Ciudad del Cabo: David Philip.

MORAÑA, Isabel. 2005. Ideologies of Hispanism. Nashville: Venderbilt University Press.

MoreTti, Franco. 2000. "Conjectures on World Literature». New Left Review 1: 54-68.

PÉREz IsAsI, Santiago. 2017. "Los estudios ibéricos como estudios literarios: algunas consideraciones teóricas y metodológicas». En Procesos de nacionalización e identidades en la península ibérica, editado por César Rina Simón, 347-361. Cáceres: Universidad de Extremadura.

PundaY, Daniel. 2003. Narrative bodies: toward a corporeal narratology. Nueva York: Palgrave Macmillan.

Robinson, Olivia. En prensa. "Performance-based Research in the Medieval Convent. European Medieval Drama 22.

Ruiz-García, Elisa. 2004. Los libros de Isabel la Católica: arqueología de un patrimonio escrito. Salamanca: Instituto de Historia del Libro y de la Lectura.

ScotT, Joan W. 2008. Género e historia, traducido por Consol Vilà I. Boadas México: Fondo de Cultura Económica. 